DiRECTEUR DE LA PUBLICATION / PUBLICATION DIRECTOR: Bruno David,

Président du Muséum national d'Histoire naturelle

RÉDACTEUR EN CHEF / EDITOR-IN-CHIEF: Jean-Philippe Siblet

ASSISTANTE DE RÉDACTION / ASSISTANT EDITOR: Sarah Figuet (naturae@mnhn.fr)

Mise EN PAGE / PAGE LAYOUT: Sarah Figuet

COMITÉ SCIENTIFIQUE / SCIENTIFIC BOARD:

Luc Abbadie (UPMC, Paris)

Luc Barbier (Parc naturel régional des caps et marais d'Opale, Colembert)

Aurélien Besnard (CEFE, Montpellier)

Vincent Boullet (Expert indépendant flore/végétation, Frugières-le-Pin)

Hervé Brustel (École d'ingénieurs de Purpan, Toulouse)

Patrick De Wever (MNHN, Paris)

Thierry Dutoit (UMR CNRS IMBE, Avignon)

Éric Feunteun (MNHN, Dinard)

Romain Garrouste (MNHN, Paris)

Grégoire Gautier (DRAAF Occitanie, Toulouse)

Olivier Gilg (Réserves naturelles de France, Dijon)

Frédéric Gosselin (Irstea, Nogent-sur-Vernisson)

Patrick Haffner (PatriNat, Paris)

Frédéric Hendoux (MNHN, Paris)

Xavier Houard (OPIE, Guyancourt)

Isabelle Leviol (MNHN, Concarneau)

Francis Meunier (Conservatoire d'espaces naturels - Hauts-de-France, Amiens)

Serge Muller (MNHN, Paris)

Francis Olivereau (DREAL Centre, Orléans)

Laurent Poncet (PatriNat, Paris)

Nicolas Poulet (OFB, Vincennes)

Jean-Philippe Siblet (PatriNat, Paris)

Laurent Tillon (ONF, Paris)

Julien Touroult (PatriNat, Paris)

COUVERTURE / COVER:

Trocheta taunensis Grosser, 2015 - Étables (Haute-Savoie), juillet 2013. Récolte et photographie: B. Lecaplain.

Naturae est une revue en flux continu publiée par les Publications scientifiques du Muséum, Paris

Naturae is a fast track journal published by the Museum Science Press, Paris

Les Publications scientifiques du Muséum publient aussi / The Museum Science Press also publish:

Adansonia, Zoosystema, Anthropozoologica, European Journal of Taxonomy, Geodiversitas, Cryptogamie sous-sections Algologie, Bryologie, Mycologie, Comptes Rendus Palevol.

Diffusion - Publications scientifiques Muséum national d'Histoire naturelle

CP 41 - 57 rue Cuvier F-75231 Paris cedex 05 (France)

Tél. : 33 (0)1 40794805 / Fax: 33 (0)1 40793840

diff.pub@mnhn.fr / https://sciencepress.mnhn.fr

(c) Publications scientifiques du Muséum national d'Histoire naturelle, Paris, 2021

ISSN (électronique / electronic): 1638-9387 


\section{Sur la présence en France de Trocheta taunensis Grosser, 2015 (Hirudinida, Erpobdellidae)}

Benoît LECAPLAIN

La Saudrais, F-35610 Sains (France)

benlecaplain@yahoo.fr

Soumis le 15 décembre 2020 | Accepté le 12 février 2021 | Publié le 1er décembre 2021

MOTS CLÉS

Sangsues,

taxonomie,

Faune de France.

KEY WORDS

Leeches,

Taxonomy,
Lecaplain B. 2021. - Sur la présence en France de Trocheta taunensis Grosser, 2015 (Hirudinida, Erpobdellidae). Naturae 2021 (25): 345-349. https://doi.org/10.5852/naturae2021a25

RÉSUMÉ

Depuis les années 1950, une espèce de sangsue du genre Trocheta est citée de France et désignée comme Trocheta bykowskii Gedroyć, 1913. Des travaux de Košel en 2004 puis de Grosser en 2015 sur les populations de l'est de l'Europe ont permis de mieux connaître la taxonomie de ce taxon qui s'est avéré être un synonyme junior de Trocheta cylindrica Örley, 1886. Cette espèce, dont la répartition est restreinte aux Carpates, n'est de fait pas présente en Europe de l'Ouest. L'espèce unicolore désignée comme bykowskii à l'ouest de l'Europe a été récemment décrite sous le nom de Trocheta taunensis Grosser, 2015. Cet article montre que les exemplaires français sont à rattacher à cette sangsue. Une clé d'identification des espèces du genre Trocheta présents en France est proposée.

\section{ABSTRACT}

On the presence in France of Trocheta taunensis Grosser, 2015 (Hirudinida, Erpobdellidae).

Since the 1950s, a leech species of the genus Trocheta has been cited from France and named Trocheta bykowskii Gedroyć, 1913. Work by Košel in 2004 and then by Grosser in 2015 on the populations of eastern Europe made it possible to better understand the taxonomy of this taxon which turn out to be a junior synonym of Trocheta cylindrica Örley, 1886. This species, whose distribution is restricted to the Carpathians, is in fact not present in Western Europe. The unicoloured species named bykowskii from these western countries has been described under the name Trocheta taunensis Grosser, 2015. Investigation of leeches named Trocheta bykowskii from France showed that these related to the newly described species T. taunensis and not to T. cylindrica. The main difference lies in examining the reproductive system and in particular the atrium, which is very elongated in taunensis. The data of T. bykowskii from France are now to be linked to the species T. taunensis. Currently, four species of the genus Trocheta have been recorded in France, T. subviridis and T. pseudodina are certainly present throughout France. T. falkneri is a leech endemic to the Pyrenees. The geographical distribution of T. taunensis was established thanks to our field observations as well as the analysis of the bibliography available on the subject. The species has been recorded in eastern France, from the departments of Drôme in the south, to the Doubs in the north and the Rhône in the west. An identification key for species of the genus Trocheta present in France is presented. 


\section{INTRODUCTION}

En 1886, Örley décrit Trocheta cylindrica Örley, 1886 de l'actuelle Slovaquie (Örley 1886). En 1888, Apáthy rejette ce taxon et le considère comme synonyme d'Erpobdella octoculata (Linnaeus, 1758) (Apáthy 1888). Le nom de Trocheta cylindrica est ensuite oublié (Košel 2004). En 1913, Gedroyć décrit, de l'actuelle Ukraine, une sangsue qu'il nomme Trocheta bykowskii Gedroyć, 1913 (Gedroyć 1913). Pendant très longtemps, jusqu'à la description de nouvelles espèces du genre Trocheta, T. bykowskii et T. subviridis Dutrochet, 1817 étaient considérés comme les seules sangsues du genre Trocheta présentes en Europe (Sawyer 1986).

Plusieurs populations de sangsues nommées T. bykowskii ont été découvertes en Europe centrale et de l'Ouest (e.g. Dresscher \& Engel 1955; Mann 1959; Bouvet 1967; Sket 1968; Nesemann 1993, 1997).

Sket (1968) suggère le premier que T. bykowskii représente en fait un complexe d'espèces. Nesemann (1990) conclut que cette espèce est restreinte au sud-est de l'Europe et que les populations découvertes en Europe occidentale correspondent à une nouvelle espèce qu'il nomme Trocheta pseudodina Nesemann, 1990. S'il est maintenant admis que les mentions de T. bykowskii de Grande-Bretagne et des Pays-Bas notamment correspondent bien à cette espèce (Van Haaren et al. 2004; Elliott \& Dobson 2015), certaines populations de France et d'Allemagne par exemple, ne correspondent pas à la description de T. pseudodina donnée par Nesemann.

Par ailleurs, le travail de Košel (2004) aura permis de mieux connaître T. cylindrica et T. bykowskii. Ce dernier est désormais considéré comme synonyme junior de T. cylindrica, espèce restreinte à l'est de l'Europe.

Une population allemande, identifiée par Nesemann (1993) comme T. bykowskii, a été analysée par Grosser (2015). Cette espèce, distinguée de T. bykowskii (= T. cylindrica), a été nommée Trocheta taunensis Grosser, 2015.

En France, une espèce du genre Trocheta a été identifiée dans plusieurs régions du sud-est et nommée T. bykowskii (e.g. Bouvet 1967; Turquin 1984). La description des sangsues dans les publications permet d'écarter T. pseudodina qui est sombre avec une série de taches claires sur chaque anneau, tandis que la description originale de $T$. bykowskii $(=T$. cylindrica $)$ présente une sangsue à coloration uniforme. Les spécimens identifiés comme T. bykowskii de France ne peuvent donc, contrairement à la Grande-Bretagne et aux Pays-Bas, être rattachés à T. pseudodina. Il était donc fort probable que ceux-ci correspondent à la nouvelle espèce $T$. taunensis.

Des spécimens récoltés en 2013 en Savoie et Haute-Savoie (Lecaplain, non publié) nous ont conduit à une analyse plus poussée pour déterminer l'identité de cette sangsue.

\section{MATÉRIEL ET MÉTHODE}

En juillet 2013, six sangsues du genre Trocheta ont été récoltées sous les pierres, dans des ruisseaux et dans une source sur les communes de Rumilly (Haute-Savoie), Étable (ancienne com-

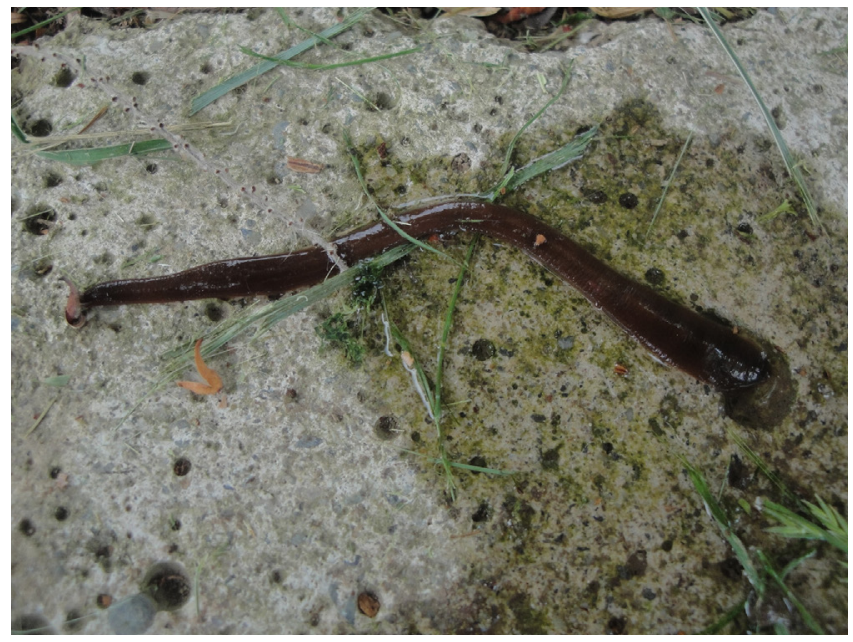

FIG. 1. - Trocheta taunensis Grosser, 2015 -Étables (Haute-Savoie), juillet 2013. Récolte et photographie: B. Lecaplain.

mune qui a fusionné en 2019 avec La Rochette pour former la commune nouvelle de Valgelon-La Rochette en Savoie) et Bourget-en-Huile (Savoie). L'analyse sous la loupe binoculaire a montré des différences par rapport à la description faite de T. bykowskii (= T. cylindrica). Plusieurs spécimens ont été envoyés à Serge Utevsky, spécialiste ukrainien des sangsues, car l'observation de l'appareil reproducteur avait montré de nettes différences avec celui de Trocheta bykowskii $(=T$. cylindrica $)$, espèce qui était alors citée de la faune de France.

Ce n'est qu'en 2019, après une nouvelle expertise des spécimens français, que Serge Utevsky et moi-même avons conclu qu'il s'agissait bien de T. taunensis.

Les exemplaires récoltés ont été déposés directement dans des tubes remplis d'éthanol à $70 \%$.

\section{SySTÉMATIQUE}

Ordre HIRUDINIDA Siddall, Apakupakul, Burreson, Coates, Erséus, Gelder, Källersjö \& Trapido-Rosenthal, 2001 Famille ERPOBDELLIDAE Blanchard, 1894

Genre Trocheta Dutrochet, 1817

Trocheta taunensis Grosser, 2015

\section{MATÉRIEL EXAMINÉ}

Cinq individus en provenance de France:

- deux individus déposés au Muséum national d'Histoire naturelle $(\mathrm{MNHN})$ : Rumilly, département de Haute-Savoie, 10 juillet 2013, Benoît Lecaplain (MNHN HEL1364); - deux individus déposés dans la collection Benoît Lecaplain (Coll. BL) : Bourget-en-Huile, département de la Savoie, 10 juillet 2013, Benoît Lecaplain;

- un individu déposé dans la Coll. BL: Étables, département de Savoie, 09 juillet 2013, Benoît Lecaplain.

\section{PrÉSENTATION DE TROCHETA TAUNENSIS}

Les spécimens analysés par Grosser (2015) avaient une longueur maximale de 114 millimètres. Les individus observés en France en 2013 n'ont pas été mesurés vivants. Les cinq sang- 


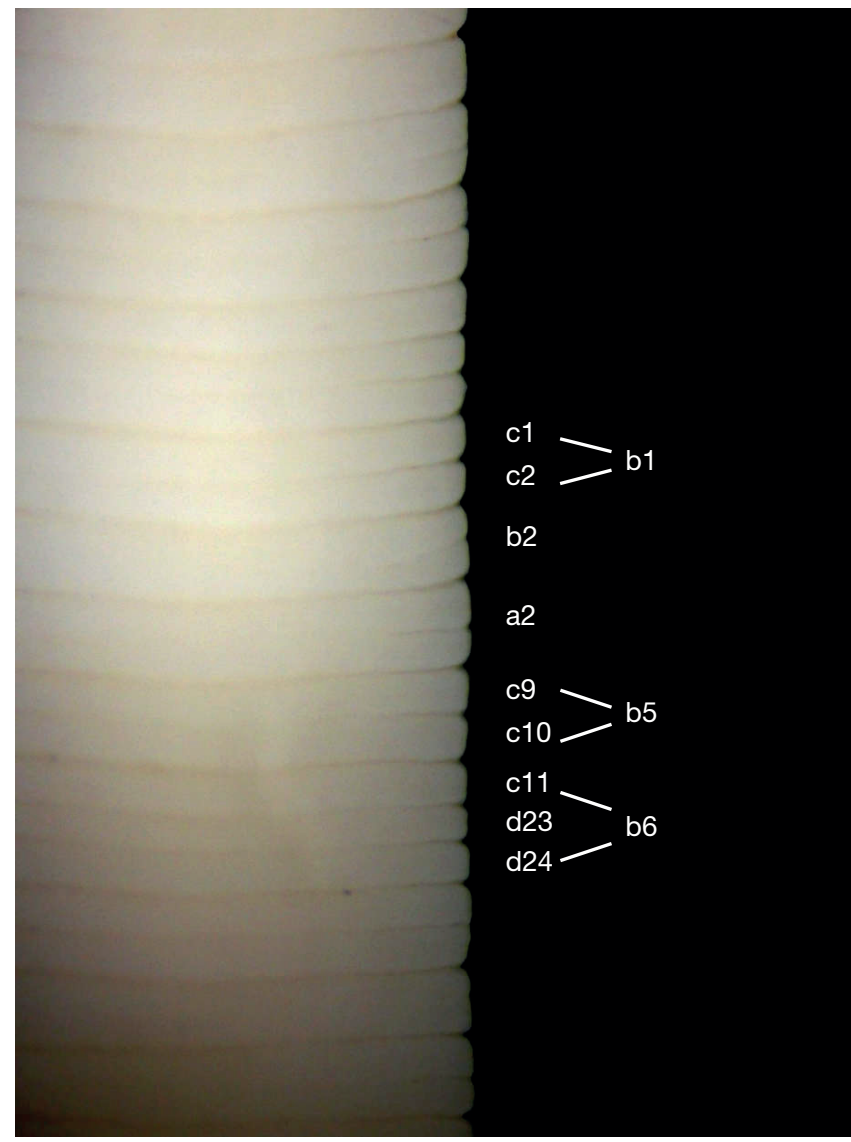

FIG. 2. - Structure des anneaux de Trocheta taunensis Grosser, 2015 - Rumilly (Haute-Savoie), juillet 2013. Récolte et photographie: B. Lecaplain.

sues conservées en alcool (collection: BL et MNHN) - et donc vraisemblablement rétractés - mesurent entre 42 et 90 millimètres. Vivante, l'espèce est brune, parfois plus foncée sur la partie antérieure (Fig. 1). Les individus en alcool sont unicolores, pâles.

Ces caractéristiques morphologiques distinguent Trocheta taunensis des autres espèces du genre Trocheta appartenant à la faune de France: T. pseudodina est sombre et possède des taches claires sur chaque anneau, T. falkneri Nesemann \& Neubert, 1996, certainement endémique des Pyrénées, présente deux lignes sombres paramédianes entourant une bande claire médiane et T. subviridis qui est plutôt verdâtre, présente généralement deux lignes longitudinales paramédianes sombres.

La structure des anneaux est typique du genre Trocheta: des anneaux simples et plusieurs anneaux doubles sur chaque somite. Grosser (2015) indique la présence de trois anneaux larges "doubles» (b2, a2, b5) et cinq anneaux étroits «simples» (c1, c2, c11, d23, d24) (Fig. 2).

Chez T. taunensis, le gonopore mâle est séparé du gonopore femelle par deux anneaux (Fig. 3) ce qui est également le cas chez pseudodina (quatre pour T. falkneri, supérieur à quatre anneaux chez subviridis). Parfois, chez taunensis, l'anneau b5 est subdivisé en c9 et c10 comme c'est le cas dans la Figure 2. Les gonopores semblent alors séparés par non pas deux mais trois anneaux. L'appréciation du nombre d'anneaux entre les

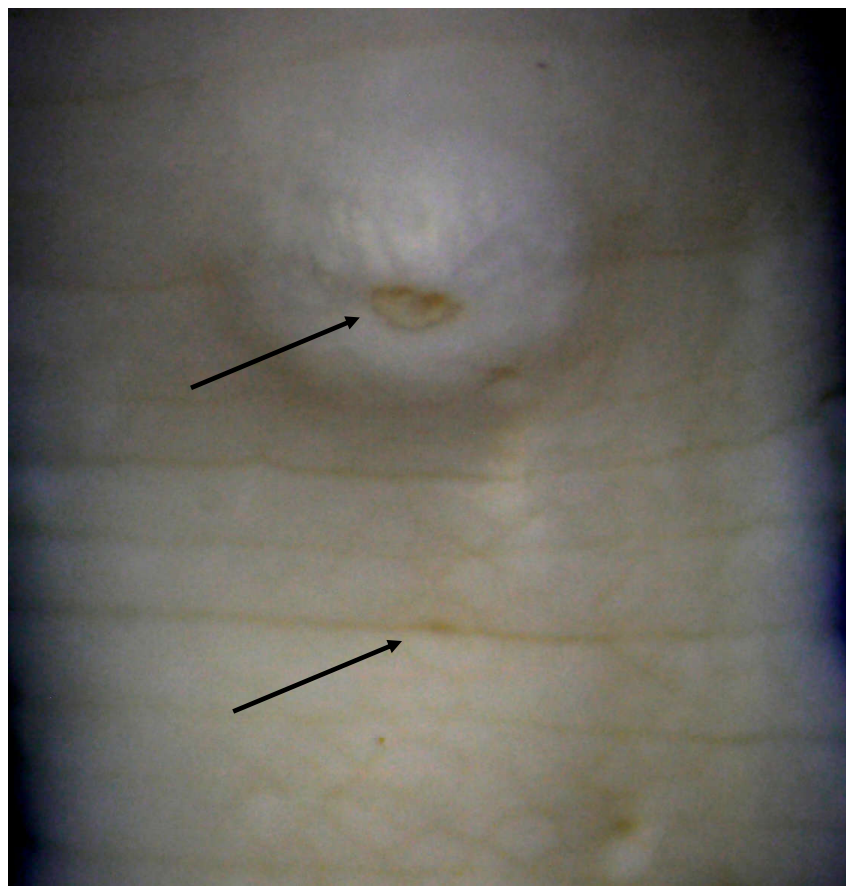

FIG. 3. - Gonopore mâle (en haut) et gonopore femelle (en bas) de Trocheta taunensis Grosser, 2015 - Rumilly (Haute-Savoie), juillet 2013. Récolte et photographie: B. Lecaplain

gonopores est parfois délicate. Il est souvent nécessaire de regarder sur plusieurs individus.

La distinction avec $T$. cylindrica se fait grâce à l'examen de l'atrium (Fig. 4). Chez T. taunensis, celui-ci est très allongé et l'extrémité antérieure se prolonge au delà du somite contenant les gonopores. Chez $T$. cylindrica, l'atrium est court, rapidement courbé, très anguleux.

\section{RÉPARTITION EN FRANCE}

L'examen de la bibliographie française disponible (Perret 1952; Bouvet 1967; Turquin 1984) a permis d'établir une carte de répartition provisoire de l'espèce. Les données ont été intégrées dans "CardObs», l'outil de saisie en ligne de données du Muséum national d'Histoire naturelle. À chaque fois, l'identification a été vérifiée afin d'écarter les données de T. pseudodina nommées T. bykowskii (sensu lato) comme cela a été le cas en Grande-Bretagne et aux Pays-Bas. Trente-deux données ont ainsi été saisies. La grande majorité de ces données (25) provient du travail réalisé par Marie-José Turquin (1984) et ses collègues sur les sangsues cavernicoles.

La localité type de Trocheta taunensis est située en Allemagne à proximité de la Belgique et du Luxembourg (Grosser 2015). De nouvelles analyses doivent être réalisées dans les localités où T. bykowskii (sensu lato) est citée en Europe de l'Ouest afin de parfaire sa répartition. Sa présence en Italie semble être confirmée par la description de T. bykowskii de Fauna d'Italia - Hirudinea (Minelli 1979). Le travail de Jueg (2008) sur les sangsues de la péninsule ibérique ne permet pas, en revanche, d'attribuer avec certitude le transfert de T. bykowskii (sensu lato) à T. taunensis.

La carte suivante (Fig. 5), réalisée à l'aide de données bibliographiques et de données personnelles, permet d'esquisser 


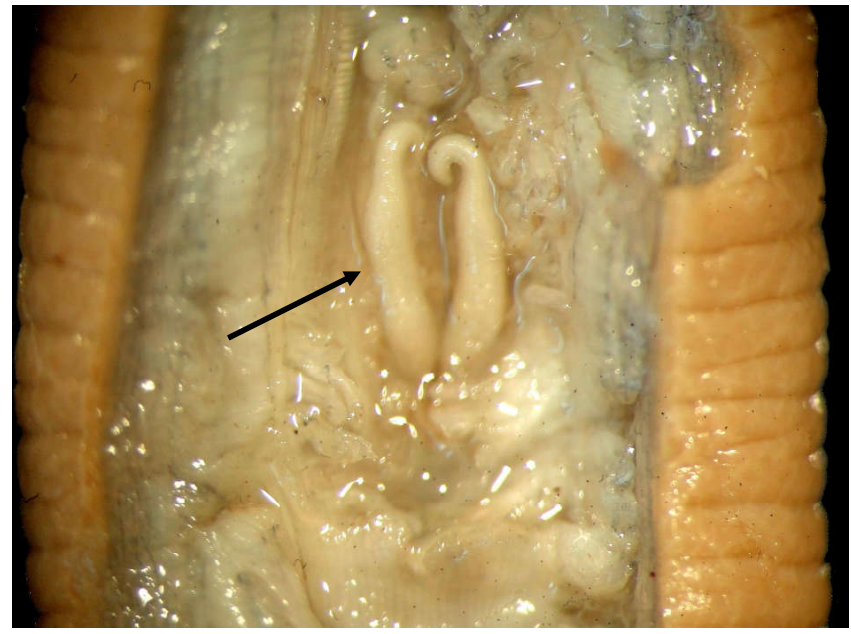

FIG. 4. - Détail de l'atrium (signalé par une flèche) de Trocheta taunensis Grosser, 2015 - Rumilly (Haute-Savoie), juillet 2013. Récolte et photographie: B. Lecaplain.

une répartition de l'espèce en France métropolitaine telle qu'actuellement connue.

Peut-être absente du pourtour méditerranéen, T. taunensis pourrait être présente dans les massifs montagneux du centre et de l'est de la France mais également dans les régions de plaines, dans les rivières ou les sources. Sa présence dans l'Ouest et le Nord est peu probable. L'espèce est à rechercher dans les Pyrénées.

\section{PROPOSITION D'UNE CLÉ D'IDENTIFICATION DES ESPÈCES FRANÇAISES DU GENRE TROCHETA}

Il n'existe actuellement aucune clé en français proposant une identification de l'ensemble des espèces du genre Trocheta observées en France. Le travail de Hondt \& Ben Ahmed (2009) sur les sangsues de France permet une approche bibliographique de l'hirudinologie française et apporte de nombreux critères d'identification. Toutefois, toutes les espèces ne sont pas mentionnées et seulement deux Trocheta sont cités.

Une clé simplifiée du genre Trocheta en France est proposée ci-après. Les genres voisins de la famille des Erpobdellidae (Erpobdella et Dina) sont évoqués tout comme le genre Barbronia, de la famille des Salifidae, proche morphologiquement des Erpobdellidae. La consultation d'une bibliographie spécifique est nécessaire en complément afin d'identifier les espèces de ce genre parfois délicat (e.g. Nesemann 1993; Grosser 2015; Lecaplain \& Noël 2019). Par ailleurs, il pourrait exister d'autres espèces de ce genre, en France, il convient donc d'être prudent dans l'identification des espèces du genre Trocheta, notamment dans les régions montagneuses.

Présence de pores accessoires

Présence de deux pores accessoires ventraux en plus des gonopores mâles et femelles...Barbronia spp.

Absence de pores accessoires

Tous les anneaux sont simples

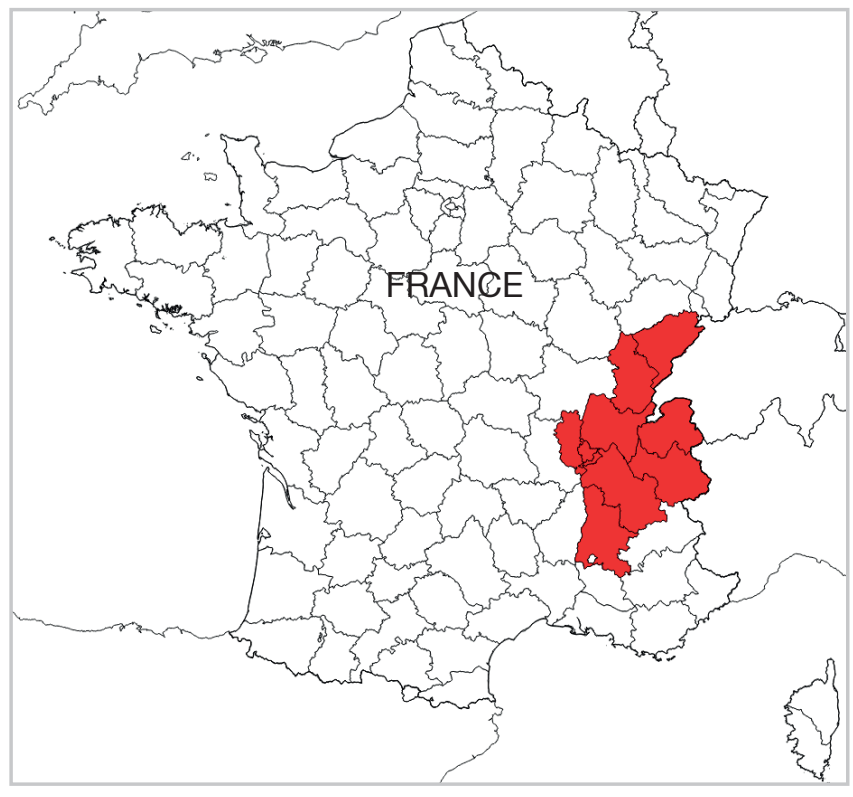

FIG. 5. - Répartition départementale avérée de Trocheta taunensis Grosser, 2015 en France.

Présence d'un anneau double et de quatre anneaux simples dans chaque somite ........................ Dina spp.

Sur chaque somite, plusieurs anneaux doubles et anneaux simples Trocheta spp. :

1. Présence sur le dos de taches rondes et claires sur chaque anneau, en une ou deux rangées, gonopores séparés par deux anneaux ........................... Trocheta pseudodina (espèce sans doute assez rare, certainement présente dans toute la France).

1'. Absence de taches claires sur le dos, parfois, présence de très nombreuses et petites papilles de même couleur que le dos....

2. Gonopores séparés par plus de quatre anneaux, verdâtre avec deux lignes paramédianes sombres (parfois absentes); souvent rougeâtre sur le ventre

Trocheta subviridis (espèce sans doute assez commune dans toute la France).

2'. Gonopores séparés par quatre anneaux ou moins ........ 3

3. Gonopores séparés par quatre anneaux, une bande médiane claire entourée de deux lignes sombres ........ Trocheta falkneri (espèce certainement endémique des Pyrénées).

3'. Gonopores séparés par deux anneaux, parfois trois ou quatre lorsque les anneaux sont subdivisés; brun lorsque l'animal est vivant, clair (crème) en alcool; absence de taches claires ainsi que de bandes ou lignes claires ou sombres sur le dos Trocheta taunensis

(espèce sans doute assez commune, actuellement connue dans l'est de la France, à rechercher ailleurs).

\section{CONCLUSION}

L'examen d'individus appartenant au genre Trocheta provenant de France a permis de confirmer la présence de T. taunensis. Les mentions antérieures de T. bykowskii doivent désormais 
être réattribuées à $T$. taunensis. La recherche de l'espèce dans les milieux favorables (sources, ruisseaux, rivières) permettra certainement de compléter la carte de répartition. L'inventaire des sangsues d'eau douce de France n'est pas figé, il reste encore de nombreuses découvertes à réaliser, de statuts à actualiser ou affiner voire d'espèces à décrire.

\section{Remerciements}

Mes remerciements vont à Serge Utevsky (Ukraine) pour ses précieux conseils et pour l'aide à l'identification de cette sangsue, ainsi qu'à Franck Noël pour la relecture de cet article. Merci à Pierre Noël et Patrick Scaps, rapporteurs, pour leurs contributions à l'amélioration de cet article.

\section{RÉFÉRENCES}

APATHY S. 1888. - Süsswasser-Hirudineen. Ein systematischer Essay. Zoologische Jahrbücher 3: 725-794.

BouveT J. 1967. - Notes sur les Hirudinées des Alpes françaises. Trochaeta bykowskii Gedroyć 1913 (sous-ordre des Pharyngobdellae, famille des Erpobdellidae). Travaux du Laboratoire d'hydrobiologie et de pisciculture de l'université de Grenoble 57-58: 105-109.

DressCHER T. G. \& ENGEL H. 1955. - Trocheta bykowskii Gedroyć, 1912 in the Netherlands. Beaufortia 5 (43): 11-13.

ELLIOTT J. M. \& DobSON M. 2015. - Freshwater Leeches of Britain and Ireland: Keys to the Hirudinea and a Review of their Ecology. Freshwater Biological Association, Ambleside, 108 p.

GedroyC M. 1913. — Zur Kenntnis der europäischen Hirudineenarten. Bulletin international de l'Académie des Sciences de Cracovie B: $32-47$

Grosser C. 2015. - Differentiation of some similar species of the subfamily Trochetinae (Hirudinida: Erpobdellidae). Ecologica Montenegrina 2 (1): 29-41. https://doi.org/10.37828/em.2015.2.3

D'HONDT J.-L. \& BEN AHMED R. 2009. —Catalogue et clés tabulaires de détermination des Hirudinées d'eau douce de la faune française. Bulletin de la Société zoologique de France 134 (3-4): 263-298.
JUEG U. 2008. — Beitrag zur Egelfauna (Hirudinea) der Iberischen Halbinsel. Lauterbornia 65: 83-104.

KošEL V. 2004. - Taxonomical position of two species of Trocheta (Hirudinea) described from Central Europe. Biologia 59 (15): $25-28$.

LECAPLAIN B. \& Nö̈L F. 2019. - Les sangsues d'eau douce du Nord-Ouest de la France (Annelida - Hirudinida) - Normandie, Bretagne, Pays de la Loire - Recherche, récolte et identification. GRETIA, PatriNat AFB-CNRS-MNHN, Paris, 92 p.

ManN K. H. 1959. - On Trocheta bykowskii Gedroyć, 1913, a leech new to the British fauna, with notes on the taxonomy and ecology of other Erpobdellidae. Proceedings of the Zoological Society of London 132 (3): 369-379.

Minelli A. 1979. - Hirudinea. Fauna d'Italia. Calderini, Bologna, $152 \mathrm{p}$.

NeSEMANN H. 1990. — Die semiaquatischen Egel mitteleuropäischer Fließgewässer (Hirudinea: Erpobdellidae, Hirudinidae) Zeitschrift fur Angewandte Zoologie 77 (2): 219-252.

NESEMANN H. 1993. - Bestimmungsschlüssel für mitteleuropäische Egel der Familie Erpobdelidae Blanchard 1894 (Hirudinea). Lauterbornia 13: 37-60.

Nesemann H. 1997. - Egel und Krebsegel (Clitellata: Hirudinea, Branchiobdellida) Österreichs. Ersten Vorarlberger Malakologischen Gesellschaft, Rankweil, Österreich, 104 p.

ÖRLEY L. 1886. - A magyarországi pióczák faunája - Fauna Hirudinearum Hungariae. Mathematikai és Természettudományi Közlemények 22: 61-115.

Perret J. L. 1952. - Cystobranchus respirans Troschel, dans le lac de Neuchâtel, et observations sur le genre Trochaeta Dutrochet. Revue Suisse de Zoologie 59: 579-583.

SAWYER R. T. 1986. - Leech Biology and Behaviour I: Anatomy Physiology, and Behavior. Vol. II Feeding, Biology, Ecology and Systematics. Clarendon Press, Oxford, 375 p.

SKeT B. 1968. — K Poznavanju Favne Pijavk (Hirudinea) v Jugoslaviji, Zur Kenntnis der Egel-Fauna (Hirudinea) Jugoslawiens. Academia Scientiarum et Artium Slovenica Classis IV: Historia Naturalis et Medicina 9 (4): 127-197.

TuRQuin M. J. 1984. — Les Hirudinées carvernicoles. Mémoires de biospéologie 11: 233-241.

Van Haaren T., Hop P., Soes M. \& Tempelman D. 2004. - The freshwater leeches (Hirudinea) of the Netherlands. Lauterbornia 52: 113-131. 